\title{
Inhibition of colorectal cancer liver metastasis in BALB/c mice following intratumoral injection of oncolytic herpes simplex virus type 2 for the induction of specific antitumor immunity
}

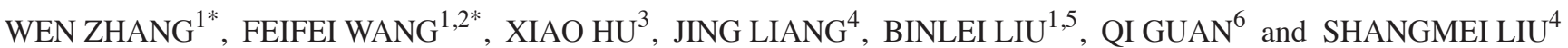 \\ ${ }^{1}$ Department of Immunology, National Cancer Center/National Clinical Research Center for Cancer/Cancer Hospital, \\ Chinese Academy of Medical Sciences and Peking Union Medical College, Beijing 100021; \\ ${ }^{2}$ Inner Mongolia University For Nationalities, Tongliao, Inner Mongolia 028000; ${ }^{3}$ State Key Laboratory of Molecular \\ Oncology; ${ }^{4}$ Department of Pathology, National Cancer Center/National Clinical Research Center for Cancer/Cancer Hospital, \\ Chinese Academy of Medical Sciences and Peking Union Medical College, Beijing 100021; \\ ${ }^{5}$ Hubei Provincial Cooperative, Innovation Center of Industrial Fermentation, Hubei University of Technology, \\ Wuhan, Hubei 30068; ${ }^{6}$ Department of Hematology and Oncology, The Second Clinical Medical School of \\ Inner Mongolia University for Nationalities, Yakeshi, Inner Mongolia 022150, P.R. China
}

Received December 5, 2017; Accepted September 5, 2018

DOI: $10.3892 / 01.2018 .9720$

\begin{abstract}
Liver metastasis represents the most prominent metastasis of colorectal cancer (CRC) and is the leading cause of CRC mortality, making the early prevention of this event very important. While current CRC therapies include surgery, radiotherapy and chemotherapy, no effective treatment option for CRC liver metastasis (CRLM) exists. Furthermore, the effects of currently available metastatic CRC drugs are frequently limited by their toxicity and side effects. Oncolytic herpes simplex virus type 2 (oHSV2) selectively infects tumor cells and also induces an antitumor immune response. The present study investigated the cytopathic effects of oHSV2 on CT-26 cells in vitro and tested its inhibitory effect on CRLM. In vitro experimental data demonstrated that oHSV2 effectively inhibited the growth of CT-26 cells. In vivo study data demonstrated that treatment with oHSV2 alone slowed the growth of subcutaneous xenograft
\end{abstract}

Correspondence to: Professor Shangmei Liu, Department of Pathology, National Cancer Center/National Clinical Research Center for Cancer/Cancer Hospital, Chinese Academy of Medical Sciences and Peking Union Medical College, 17 Panjiayuan Nanli, Beijing 100021, P.R. China

E-mail: liu_shangmei@sina.com

Professor Qi Guan, Department of Hematology and Oncology, The Second Clinical Medical School of Inner Mongolia University for Nationalities, 81 Lincheng Road, Yakeshi, Inner Mongolia 022150, P.R. China

E-mail: guanqi5818@126.com

${ }^{*}$ Contributed equally

Key words: colorectal cancer liver metastasis, oncolytic virotherapy, herpes simplex virus type 2 , antitumor immune response tumors without inducing weight loss and also inhibited CRLM by increasing the numbers of cluster of differentiation (CD) $4^{+}$ $\mathrm{T}, \mathrm{CD}^{+} \mathrm{T}$ and natural killer cells. In summary, oHSV2 shows potential as a safe and effective therapeutic agent for inhibiting the metastasis of CT-26 CRC cells to the liver.

\section{Introduction}

According to U.S. cancer statistics, colorectal cancer (CRC) is the third most common cancer and the second leading cause of cancer-associated mortality (1). The Westernized lifestyle, particularly increases in the prevalence of obesity and physical inactivity, has likely impacted the recently observed rise in CRC incidence rates in China (2,3). Furthermore, 50-60\% of patients diagnosed with CRC will develop distant metastases, which is the most common reason for CRC mortality. The most prominent CRC metastasis is that of the liver, with $80-90 \%$ of these patients having unresectable metastatic liver disease (4-7). Currently, hepatic resection of isolated CRC liver metastasis (CRLM) remains the only potential curative option for patients with this disease. Although resection is combined with modern adjuvant systemic regimens, the curative rate of CRLM is only $20 \%$, with $70 \%$ of these patients developing recurrence, primarily in the liver (8-10). Therefore, early prevention of CRLM is important. Current CRC therapeutic approaches include surgery, radiotherapy and chemotherapy. However, no effective treatments for CRLM exist, and the effects of available metastatic CRC drugs are frequently limited by their toxicity and side effects (11). By contrast, since it selectively infects tumor cells and forms syncytia, oncolytic herpes simplex virus type 2 (oHSV2) may induce antitumor immune responses (12).

HSV-2 is a common genital pathogen and prevalent sexually transmitted DNA virus (13) that has been employed as an oncolytic agent, and certain indications suggest that HSV-2 has a higher oncolytic activity compared with HSV-1. 
Zhao et al (14) constructed a novel oHSV2 agent that was able to slow tumor growth without inducing weight loss. This novel virus was demonstrated to increase the number of natural killer (NK) cells and mildly decrease the number of regulatory $\mathrm{T}$ cells (Tregs) in the spleen. The oHSV2 FusOn-H2 was shown to have a higher oncolytic activity compared with that of HSV-1. Furthermore, HSV-2 is able to induce strong $\mathrm{T}$-cell responses and inhibit primary breast cancer metastasis (15). HSV-2 provides a more effective treatment for disseminated tumors in the peritoneal cavity and for metastatic human ovarian cancer compared with HSV-1 (16). In another previous study based on infected cell protein 0 (ICP0) mutations within HSV-1 and HSV-2, ICP0-defective HSV-2 (HSV-2 dICP0) exhibited more potent antitumor activity at a lower viral burst size and induced higher levels of cytopathic effects (CPE) compared with HSV-1 dICP0 (17). The HSV-2-based oncolytic virus $\triangle \mathrm{PK}$ inhibited melanoma cells from secreting the immunosuppressive cytokine interlukin-10 and inhibited the expression of the negative immune checkpoint regulator cytotoxic $\mathrm{T}$ lymphocyte antigen 4 , which significantly increased its oncolytic activity (18). The ICP10 and $\triangle$ PK HSV-2 agents displayed oncolytic activities against melanoma via virus-induced programmed cell death pathways (19).

To the best of our knowledge, no reports on the effects of oHSV2 on an in vivo mouse CRLM model exist in the literature. In preclinical models, oHSV2 is an effective killer of CRC and CRC stem-like cells, a significant inhibitor of tumor growth and a promising therapeutic approach for patients with CRC (20). The present study aimed to assess the potential viability of oHSV2 as a therapeutic agent for the inhibition of CRLM. Construction of a CRLM model by intrasplenic injection of DX-3 or PC-3-P cell lines had revealed more marked metastatic capacity compared with the direct intravenous injection of the aforementioned cell lines (21). Here, a CRLM model was established in BALB/c mice by intrasplenically injecting CT-26 cells into the splenic capsule, and CT-26 cells were also subcutaneously injected into the right flanks of the mice. oHSV2 was administered via intratumoral injection into subcutaneous xenograft tumors in the CRLM model, to assess its therapeutic potential in CRLM and its ability to induce immune responses.

\section{Materials and methods}

Cell culture and reagents. The mouse colorectal cancer cell line CT-26 was purchased from the American Type Culture Collection (Manassas, VA, USA) and maintained in our laboratory. The cells were cultured in RPMI-1640 medium (Hyclone; GE Healthcare Life Sciences, Logan, UT, USA) supplemented with $100 \mathrm{U} / \mathrm{ml}$ penicillin, $100 \mathrm{mg} / \mathrm{ml}$ streptomycin, and $10 \%$ fetal bovine serum (FBS) (Hyclone; GE Healthcare Life Sciences) in a $5 \% \mathrm{CO}_{2}$ humidified incubator at $37^{\circ} \mathrm{C}$.

Virus. The oHSV2 used in this study is a replication-competent, genetically stable attenuated HSV-2 whose construction was previously described in detail (14). This virus was derived from the wild-type HSV-2 strain HG52 and has copy deletions of ICP34.5 and ICP47, which increases tumor selectivity and decreases virulence.
Cytotoxicity analysis of oHSV2 in CT-26 cells in vitro. CT-26 cells were plated on 6-well flat-bottom plates at $3.0 \times 10^{5}$ cells/well and grown overnight. Subsequently, the cells were infected with oHSV2 at multiplicities of infection (MOIs) of $0.1,1$ and 3 and incubated at $37^{\circ} \mathrm{C}$ in a $5 \% \mathrm{CO}_{2}$ humidified incubator. Photomicrographs of the cells were captured using an Olympus confocal microscope (Olympus Corporation, Tokyo, Japan) equipped with a Leica camera (Leica Microsystems GmbH, Wetzlar, Germany) with a x100 magnification after an additional 24, 48 and $72 \mathrm{~h}$ of culture.

Cell Counting kit 8 (CCK8) cell viability assay. The CCK8 assay (Dojindo Molecular Technologies, Inc., Kumamoto Japan) was utilized to measure cell viability. Briefly, CT-26 cells were seeded in 96-well plates at $1.0 \times 10^{4}$ cells/well in a total volume of $100 \mu \mathrm{l}$; each sample was analyzed in triplicate. To test the effect of MOI on cell viability, cells were infected with oHSV2 at MOIs of 0.1, 1 and 3. Following culturing for 24,48 , and $72 \mathrm{~h}$, the culture medium was replaced with $100 \mu \mathrm{l}$ of a solution containing $10 \% \mathrm{CCK} 8$, and the mixture was incubated for $2 \mathrm{~h}$ at $37^{\circ} \mathrm{C}$. Absorbance was measured using a Model 550 microplate reader (Bio-Rad Laboratories, Inc., Hercules, CA, USA) at $450 \mathrm{~nm}$ with a reference of $655 \mathrm{~nm}$. The untreated control was set to $100 \%$, and the treated samples were normalized to this value according to the following equation: Survival rate $(\%)=($ optical density $(\mathrm{OD})$ of the treated cells-OD of blank control/OD of negative control-OD of blank control) x100.

Animals and treatment. A total of 72 6-week-old female BALB/c mice (Animal Center of the Chinese Academy of Medical Science, Beijing, China), with a mean weight of 16-18 g, were fed chow and water ad libitum, and housed under specific pathogen-free conditions. The mice were acclimated to the housing conditions (mean temperature of $24 \pm 2^{\circ} \mathrm{C}$ and mean humidity of $52 \pm 8 \%$ ) for 7 days. The study protocol was approved by the Ethics of Animal Experiments Committee of the Chinese Academy of Medical Sciences and Peking Union Medical College (Beijing, China).

Liver metastasis animal model. The experimental CRLM model was established via intrasplenic and subcutaneous inoculation of CT-26 cells; tumor cell viability during the exponential growth phase was $>95 \%$. CT-26 cells $\left(3 \times 10^{5}\right)$ were suspended in $100 \mu \mathrm{l}$ RPMI-1640 serum-free medium (SFM) and subcutaneously inoculated into the right flanks of the mice. The mice were anesthetized via an intraperitoneal injection of $5 \%$ chloral hydrate $(400 \mathrm{mg} / \mathrm{kg}$ body weight) and fixed in the prone position. An abdominal incision $\sim 1.0 \mathrm{~cm}$ in length was made between the dorsal central and left axillary midlines to expose the spleen. Equal numbers and volumes of CT-26 tumor cells were inoculated into the splenic tip using a 4-gauge needle to produce a visible pale wheal, followed by hemostasis for 5 min with alcohol-soaked cotton balls and wound closure. The abdominal wound was closed if no significant bleeding or extravasation was encountered.

Animal experiments. When the subcutaneous xenograft tumors reached $3 \mathrm{~mm}$ in diameter, tumor volumes were 

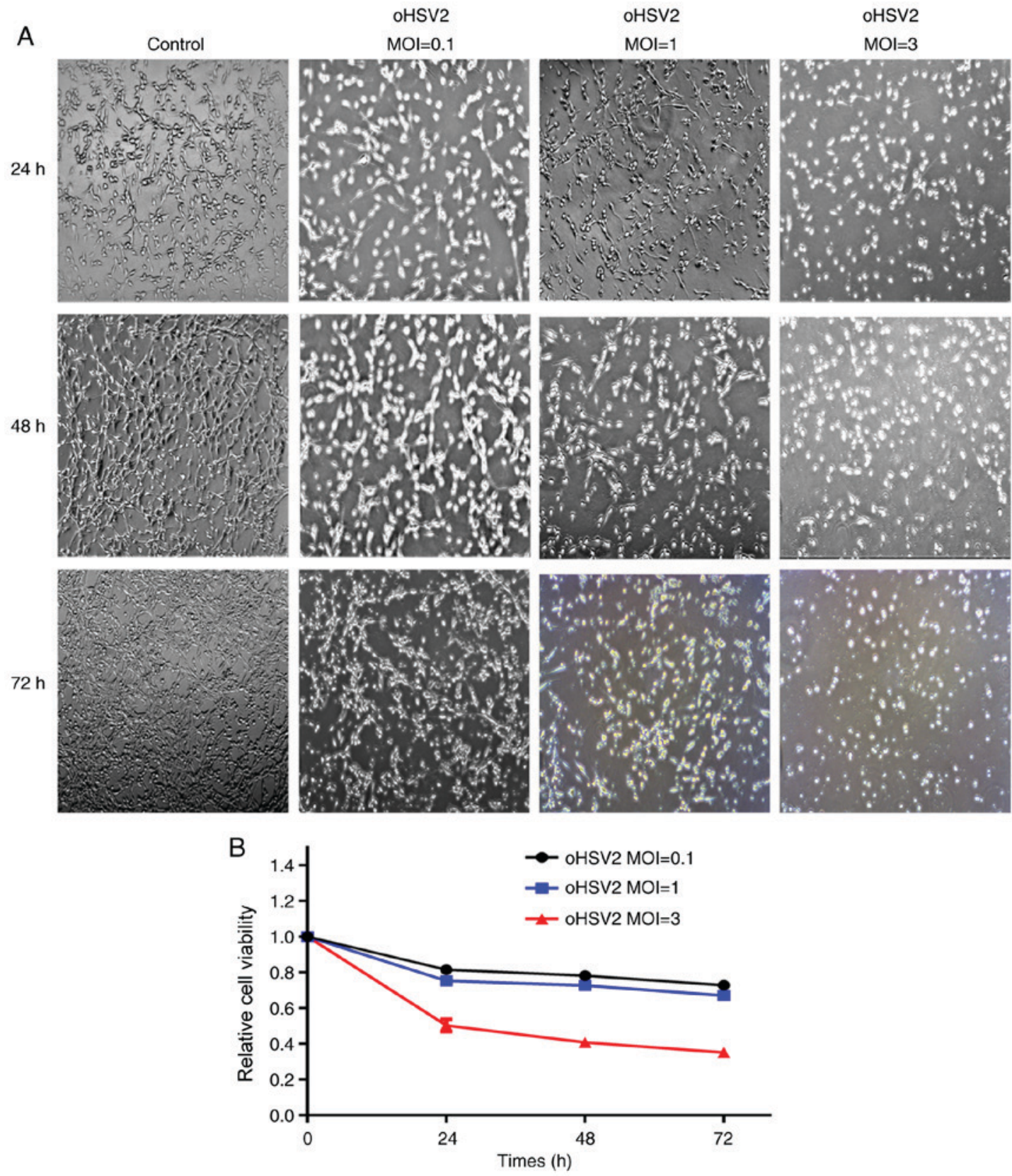

Figure 1. Oncolytic effect of oHSV2 on CT-26 cells in vitro. (A) CT-26 cells were infected with oHSV2 at the indicated MOIs and times. Cells were observed with an inverted phase contrast microscope with a x100 objective. (B) CT-26 cells were treated with oHSV2 at different MOIs for 24, 48 and 72 h. Each value represents the mean \pm standard error of three independent samples. MOI, multiplicity of infection; oHSV2, oncolytic herpes simplex virus 2.

calculated according to the following formula: Tumor volume $\left(\mathrm{mm}^{3}\right)=\left(\right.$ length $\mathrm{x}$ width$\left.{ }^{2}\right) / 2$. The mice were randomly divided into two groups as follows: i) Treatment with oHSV2 alone on days 1,3, 5, 7 and 9; and ii) control treatment with RPMI-1640 SFM on days $1,3,5,7$ and 9 . oHSV2 $\left(1 \times 10^{6} \mathrm{PFU}\right.$ in $\left.100 \mu \mathrm{l}\right)$ was applied by intratumoral injection into the right flank subcutaneous xenograft tumors. The tumor size and body weight of each mouse were measured every other day, and mouse survival was monitored daily during the experimental period. The tumor volumes were determined by caliper measurement at the indicated times after treatment. A total of 7 days after the last treatment, the mice were sacrificed by cervical dislocation and then subjected to gross dissection. Their livers and spleens were removed and weighed, and the numbers of visible tumor nodules on the liver surfaces were counted.
Histopathological analysis. Following the aforementioned procedures, the livers and spleens were collected and fixed in $10 \%$ paraformaldehyde for $12-24 \mathrm{~h}$ at room temperature then paraffin-embedded. Sections of $4 \mu \mathrm{M}$ were stained with hematoxylin and eosin at room temperature (3-5 min for hematoxylin and 5-10 sec for eosin). Samples were permeabilized with $0.1 \%$ Triton X-100, and nonspecific binding was blocked with 5\% normal goat serum (Invitrogen; Thermo Fisher Scientific, Inc., Waltham, MA, USA). The pathological sections were observed with an confocal microscope (magnification, $\mathrm{x} 400$; Olympus Corporation, Tokyo, Japan) equipped with a Leica camera (Leica Microsystems GmbH, Wetzlar, Germany).

Characterization of $C D 4^{+} T, C D 8^{+} T$ and natural killer (NK) cells in peripheral blood using flow cytometric analysis. A 
total of 2 days after the last oHSV2 treatment, $\mathrm{CD} 4^{+} \mathrm{T}, \mathrm{CD} 8^{+} \mathrm{T}$ and NK cells were quantified in mouse peripheral blood $(n=3)$. Peripheral blood lymphocytes were isolated by centrifugation at $250 \mathrm{x} \mathrm{g}$ at $4^{\circ} \mathrm{C}$ for $10 \mathrm{~min}$ in a gradient lymphocyte isolation solution for mice (Tianjin Hao Yang Biological Manufacture Co., Ltd., China) at room temperature and washed twice with PBS. The cell suspensions were then stained for $30 \mathrm{~min}$ at $4^{\circ} \mathrm{C}$ using the following monoclonal antibodies: Fluorescein isothiocyanate (FITC) anti-mouse CD8a (cat. no. 100708; dilution, 1:200), Percp/cy5.5 anti-mouse CD4 (cat. no. 100406; dilution, 1:80), Allophycocyanin anti-mouse CD3 (cat. no. 100236; dilution, 1:40), and FITC anti-mouse 49b (cat. no. 103503; dilution, 1:200; all obtained from BioLegend, Inc., San Diego, CA, USA). Following washing with PBS, the cells were fixed with $4 \%$ paraformaldehyde at room temperature for $20 \mathrm{~min}$, and the $\mathrm{CD}^{+} \mathrm{T}, \mathrm{CD} 8^{+} \mathrm{T}$ and $\mathrm{NK}$ cell frequencies were determined using flow cytometry (FACSCalibur; BD Biosciences, Franklin Lakes, NJ, USA) and analyzed by FlowJo 7.6.1 software (TreeStar, Inc., Ashland, OR, USA). The results were expressed as the relative fluorescence index by dividing the fluorescence value of the experimental groups by that of the control group.

Statistical analysis. All data were statistically analyzed in this study were performed using GraphPad Prism 6.0 (GraphPad Software, Inc., La Jolla, CA, USA). All data are expressed as the mean \pm standard error of the mean from at least three independent experiments. Independent sample t-tests were used to analyze the significance of differences between two groups. Survival curves were calculated using the Kaplan-Meier method and compared with the log-rank test. $\mathrm{P}<0.05$ was considered to indicate a statistically significant difference.

\section{Results}

oHSV2 has cytotoxic effects on CT-26 cells in vitro. To investigate the effects of oHSV 2 on CT-26 cells in vitro, cells were infected with oHSV2 at different MOIs. As presented in Fig. 1A, as the oHSV2 MOI increased, the cellular CPE became more obvious, and the distance between the cells gradually increased. This result suggested that the oncolytic effect of oHSV2 on CT-26 cells may be dependent on the MOI.

To further examine the effects of oHSV2 on CT-26 cells in vitro, CT-26 cells were infected with virus at various MOIs (MOI=0.1, 1 and 3) for 24, 48 and $72 \mathrm{~h}$ and subjected to the CCK8 assay to investigate alterations in cell viability. The viral treatment induced a concentration-dependent reduction in CT-26 cell viability. As presented in Fig. 1B, cells treated with oHSV2 at MOI=0.1 for $72 \mathrm{~h}$ had a viability of $72.79 \%$, whilst the viability decreased to $67.09 \%$ with $\mathrm{MOI}=1$ and to $35.20 \%$ with $\mathrm{MOI}=3$.

Intratumoral oHSV2 injection inhibits subcutaneous CT-26 cell xenograft tumor growth with no systemic side effects. As expected, a single oHSV2 treatment application induced significant growth inhibition compared with that induced by the control. As presented in Fig. 2A, in the oHSV2 treatment group the subcutaneous xenograft tumor volume remained approximately the same, whereas the tumor volume was significantly increased in the control group. Notably, tumor volume in the oHSV2 treatment group increased slightly during the first 6 days and began to decrease on day 8 . However, tumor volume in the control group remained higher compared with that in the oHSV2 treatment group throughout the experiment. Furthermore, alterations in the body weights of mice treated with oHSV2 compared with the control were not statistically significant (Fig. 2B), and no necrosis or skin ulcerations were observed in the oHSV2 treatment group. Two days after the last treatment, mice in the oHSV2 treatment group had glossy, healthy fur and exhibited normal behavior. In contrast, the behavior of the mice in the control group were apathetic and dull, and their fur was disordered (Fig. 2C). These results demonstrated that oHSV2 exerted significant antitumor effects and did not influence mouse body weight.

$C R L M$ in $B A L B / c$ mice. Mice were sacrificed by cervical dislocation at 19 days post-tumor cell inoculation. Their livers were dissected, and different-sized nodules were distributed on the liver surfaces of all mice (Fig. 3). Significantly more nodules of diameter $>1 \mathrm{~mm}$ were observed in the control group compared with the oHSV2 treatment group. As presented in Fig. 3, histopathological analysis of tissue sections confirmed that the liver nodules were poorly differentiated adenocarcinomas, demonstrating successful establishment of the CRLM model. The results revealed that the intrasplenic injection was effective in building the CRLM model in BALB/c mice.

oHSV2 treatment inhibits CRLM in vivo. As described above, the livers and spleens of the mice were separated and weighed, and the numbers of visible tumor nodules on the liver surfaces were counted in the two groups 7 days after the last treatment. As presented in Fig. 3, numerous tumor nodules formed on the livers of the control group mice, while few tumor nodules formed on the livers of the oHSV2 mice. Statistical analysis revealed that the number of nodules formed in the control group livers was significantly higher compared with those of the oHSV2 treatment group (Fig. 4A). As presented in Fig. 4B, liver weights in the control group were increased compared with those in the oHSV2 treatment group $(\mathrm{P}<0.05)$. However, the spleen weights in the control and oHSV2 treatment groups were not significantly different (Fig. 4C, $\mathrm{P}=0.116$ ). With the exception of the mice used in the aforementioned experiments, mouse survival was assessed every day until the end of the experiment, revealing that the overall survival (OS) of mice in the oHSV2 treatment group was prolonged compared with that of control group mice (Fig. 4D, $\mathrm{P}<0.05)$. These data illustrated that intratumoral injection of oHSV2 into the right flanks of mice with established liver metastasis nodules significantly inhibited CRLM and prolonged OS.

oHSV2 treatment increases the percentages of $C D 4^{+} T, C D 8^{+}$ $T$ and $N K$ cells in murine peripheral blood. To examine the mechanisms underlying the suppression of CRLM by treatment with oHSV2 in immunocompetent BALB/c mice bearing the CT-26 cell CRLM model, the effects of oHSV2 on inducing immunological alterations in the peripheral blood were investigated. The percentages of $\mathrm{CD}^{+} \mathrm{T}, \mathrm{CD} 8^{+}$ $\mathrm{T}$ and NK cells in mouse peripheral blood were assessed by 

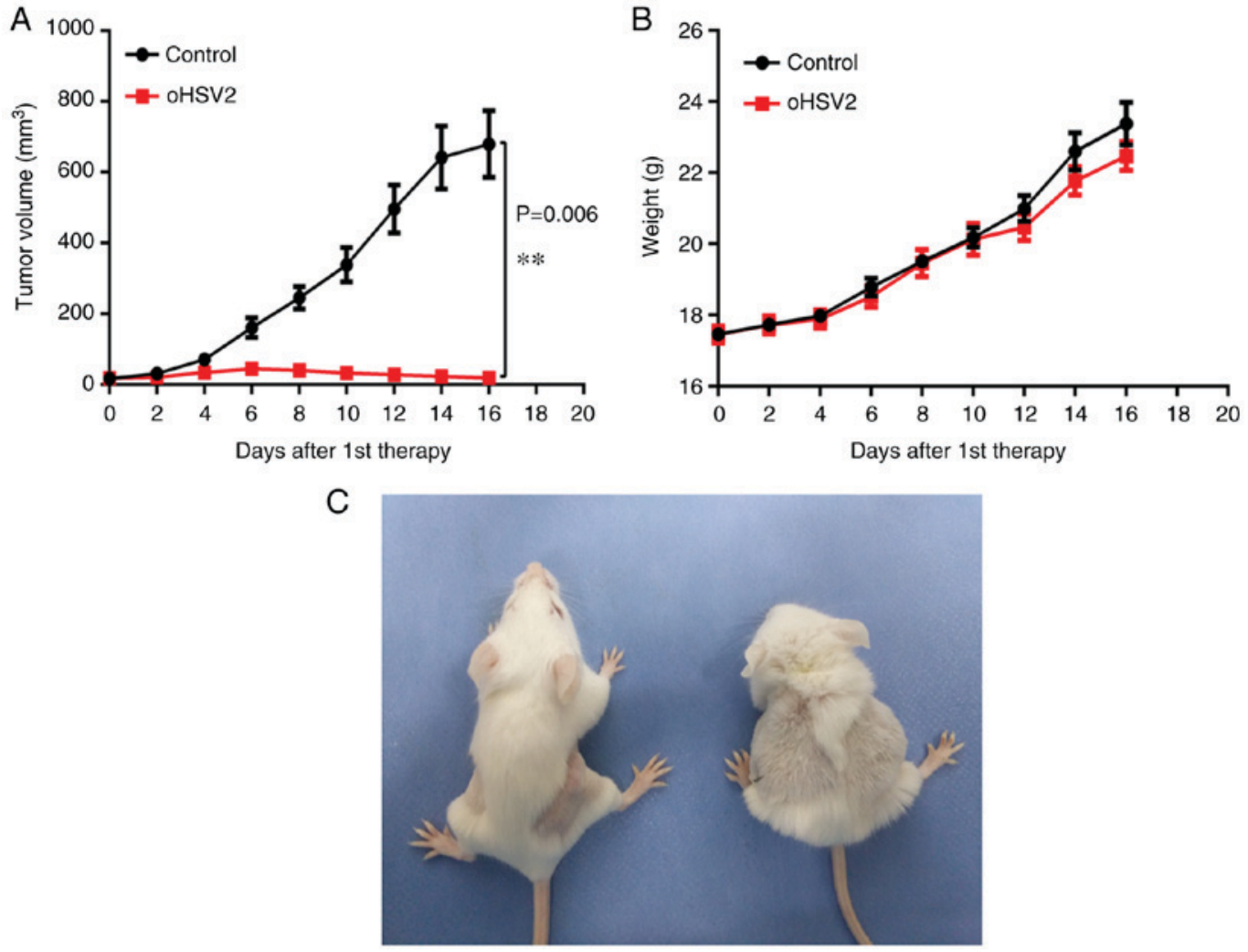

Figure 2. Effects of oHSV2 on subcutaneous xenograft tumors and mouse body weight. (A) Tumor volume was measured every 2 days following the first treatment. The data are presented as the mean $\pm \operatorname{SEM}(n=15), P=0.006$. (B) The mice were weighed every 2 days following the first treatment. The data are presented as the mean \pm SEM $(n=15)$, and the differences were not significant different. (C) Left, oHSV2 treatment group; right, control group. " $\mathrm{P}<0.01$. oSHV2, oncolytic herpes simplex virus 2; SEM, standard error of the mean.

Liver tissue
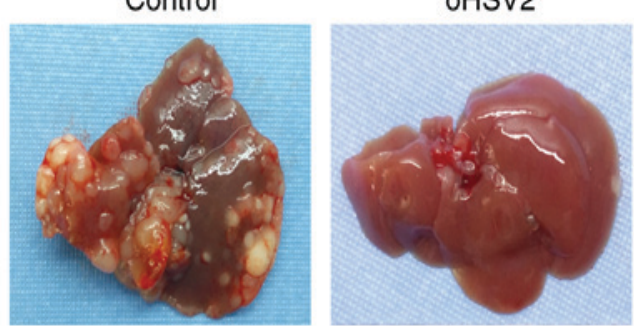

Pathological section
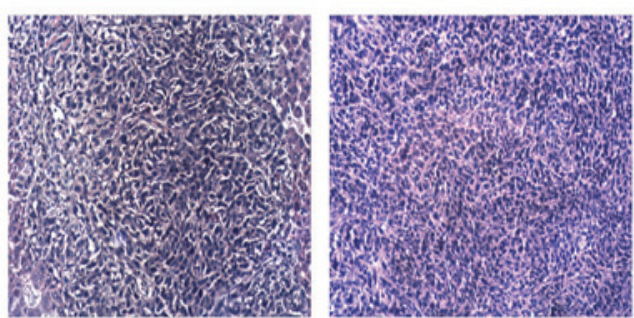

Figure 3. The appearance and pathological section of livers of mice from each group. Upper panel, representative macroscopic images of mouse livers; nodules of varying sizes were distributed on the liver surfaces. Lower panel, representative sections stained with hematoxylin and eosin demonstrating the histopathology of mouse livers in the two treatment groups. The pathological sections were observed with an inverted phase contrast microscope at magnification, $\mathrm{x} 400$.

flow cytometry. As presented in Fig. 5, treatment with oHSV2 increased the peripheral blood percentages of $\mathrm{CD} 4^{+} \mathrm{T}, \mathrm{CD} 8^{+}$ $\mathrm{T}$ and NK cells compared with those in the control group $(\mathrm{P}=0.0250,0.0025$ and 0.0166 , respectively), suggesting that
oHSV2 may upregulate specific antitumor immune responses in BALB/c mice bearing the CT-26 cell CRLM model.

\section{Discussion}

It has previously demonstrated that oHSV2 may cytolytically destroy tumor cells in vitro and inhibit xenograft tumor growth by increasing the immune response in immunocompetent mice in vivo (14,22-24). Oncolytic viruses are able to specifically target and kill tumor cells and provide an in situ cancer immunotherapy vaccine. Investigators have demonstrated that HSV vectors may inhibit the growth of established tumors at distant sites (25), although the potential inhibition of CRLM by oncolytic viruses has not been examined.

Despite recent improvements in comprehensive CRC therapies, the 5-year survival rate for metastatic CRC (mCRC) remains $<30 \%$ (26). To date, treatment with all of the available drugs in combination or in sequence until progression or unacceptable toxicity occurs has been the standard therapy for mCRC (27). Viruses are currently the most promising therapeutic approach to treating tumors $(28,29)$. In 2015, the US Food and Drug Administration approved talimogene laherparepvec (T-VEC) for use in melanoma patients with injectable, nonresectable skin and lymph node lesions (30).

Oncolytic viruses specifically infect, replicate in, and kill cancer cells while leaving normal cells unharmed $(31,32)$, and simultaneously release tumor-specific antigens that are recognized by $\mathrm{CD}^{+} \mathrm{T}$ lymphocytes (33). oHSV2 agents are 
A
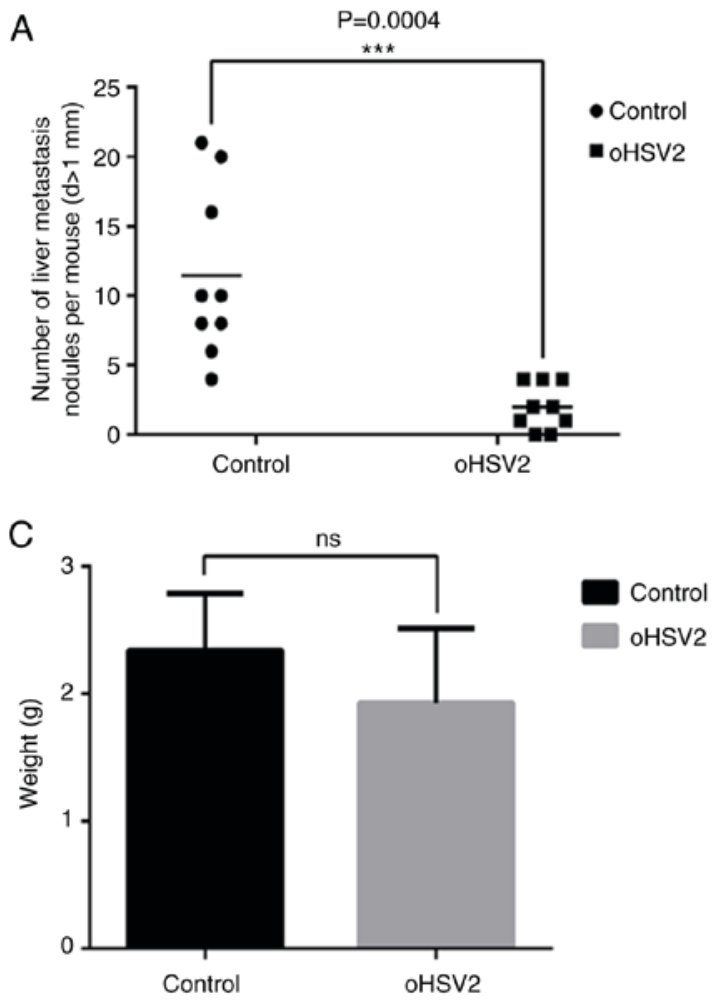
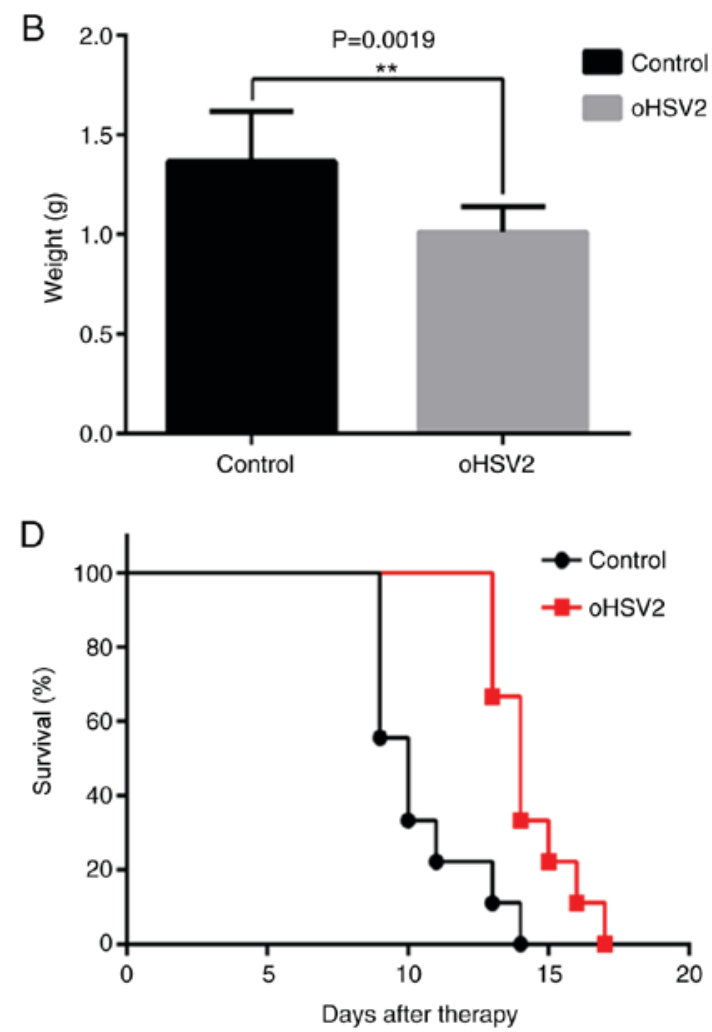

Figure 4. Therapeutic effects of oHSV2 on colorectal cancer liver metastasis. (A) Fewer liver metastasis nodules were observed in mice in the oHSV2 treatment group compared with those in control group mice. The data represent the mean \pm SEM ( $n=9, P=0.0004)$. (B) The weights of livers from mice in the oHSV2 treatment group were less than those of control group mice. The data represent the mean $\pm \mathrm{SEM}(\mathrm{n}=9, \mathrm{P}=0.0019)$. (C) The difference in spleen weight between oHSV2 treatment group and control group mice were not significantly different (ns, $\mathrm{P}=0.116$ ). (D) Kaplan-Meier survival curve comparing overall survival between the two groups $(\mathrm{n}=9)$. Median survival with oHSV2 was 14 days compared with 10 days with control treatment $(\mathrm{P}=0.0011)$. ${ }^{* * *} \mathrm{P}<0.01$ and ${ }^{* * * *} \mathrm{P}<0.001$. oHSV2, oncolytic herpes simplex virus 2; SEM, standard error of the mean.
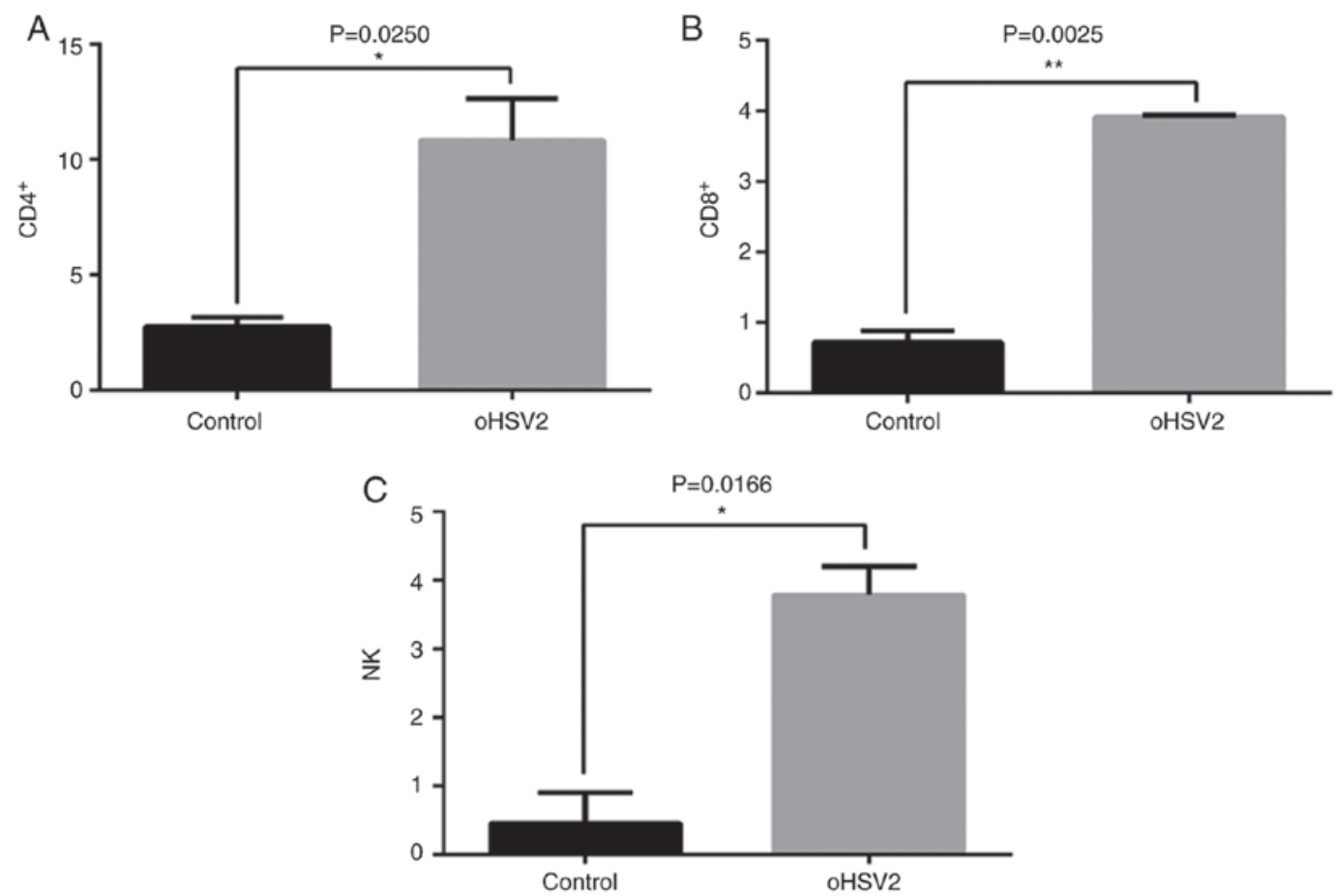

Figure 5. Treatment with oHSV2 increases the ratios of $\mathrm{CD} 4^{+} \mathrm{T}, \mathrm{CD} 8^{+} \mathrm{T}$ and $\mathrm{NK}$ cells in vivo. (A) Percentage of $\mathrm{CD} 4^{+} \mathrm{T}$ cells in peripheral blood following treatment with oHSV2 compared with the control group. Statistical analysis was performed using an unpaired Student's t-test $(\mathrm{P}=0.0250)$. (B) Percentage of $\mathrm{CD} 8^{+} \mathrm{T}$ cells in the peripheral blood following treatment with oHSV2 compared with the control group. Statistical analysis was performed using an unpaired Student's t-test $(\mathrm{P}=0.0025)$. (C) Percentage of NK cells in the peripheral blood following treatment with treatment compared with the control group. Statistical analysis was performed using an unpaired Student's t-test $(\mathrm{P}=0.0166)$. ${ }^{*} \mathrm{P}<0.05$ and ${ }^{* *} \mathrm{P}<0.01$. oHSV2, oncolytic herpes simplex virus 2 ; CD, cluster of differentiation; NK cells, natural killer cells. 
armed with granulocyte-macrophage colony-stimulating factor, a hematopoietic growth factor and pro-inflammatory cytokine that promotes the activation and tissue accumulation of monocytes, macrophages, and granulocytes $(14,34,35)$. The majority of solid tumors are infiltrated by a large variety of immune cells, including $\mathrm{CD}^{+} \mathrm{T}$ cells $\left(\mathrm{CD}^{+}{ }^{+}\right.$helper and $\mathrm{CD} 8^{+}$ cytotoxic T cells) and NK cells (36-38).

The authors of the present study have previously demonstrated the antitumor effects of oHSV2 (14). In the present study, a series of experiments were performed to further assess the therapeutic effects of oHSV2 on CRLM in mice. First, the effects of oHSV2 on CT-26 cells were assessed with a CCK8 assay in vitro, revealing that oHSV2 markedly inhibited CT-26 cell growth. In addition, as the MOI of oHSV2 increased, the survival rate of CT-26 cells gradually deceased. To evaluate whether oHSV2 was effective, a right flank subcutaneous xenograft tumor and CRLM model was successfully established in BALB/c mice and oHSV2 was intratumorally-injected into the xenograft tumors. Tumor volumes in the oHSV2 treatment group were significantly lower compared with those in the control group, and the numbers of metastasized liver nodules exhibited a similar trend, illustrating that oHSV2 was able to inhibit the growth and metastasis of the CT-26 cell CRLM model. Flow cytometry was used to analyze peripheral blood samples collected from the two groups of mice, in order to assess the effects of immune cell numbers on the inhibition of CRLM. The percentages of $\mathrm{CD}^{+} \mathrm{T}, \mathrm{CD}^{+} \mathrm{T}$ and $\mathrm{NK}$ cells in the oHSV2 treatment group were increased compared with those in the control group, demonstrating that oHSV2 delivered intratumorally to subcutaneous xenograft tumors induced immune responses to inhibit CRLM.

CRLM is a dynamic process in which the immune microenvironment serves an important role in tumor initiation, proliferation, growth and metastasis (39). However, tumors resist clinical treatment via the rapid progression of immune escape and immunosuppressive mechanisms, leading to treatment failure (40). Meanwhile, cellular immune reactions are modified during metastasis, inducing tumor cell senescence (41). In the context of oncolytic HSV, the initial stages of immunogenic virus replication leading to activation of antitumor immunity were demonstrated to be more important compared with the persistence of the virus replicating within the tumor (42), illustrating the importance of controlling the tumor microenvironment for successful tumor therapy.

To the best of our knowledge, this is the first study which evaluates the antimetastatic effects of oHSV2 in a CRLM model established in BALB/c mice. Herein, oHSV2 was intratumorally-injected into right flank subcutaneous xenograft tumors, inducing indirect inhibition of tumor growth and inhibition of CRLM. In vitro, the growth capacity of CT-26 cells was reduced by administration of oHSV2. In vivo, treatment with oHSV2 effectively prolonged the overall mouse survival and also restricted liver metastasis in the CRLM model. oHSV2 also caused an increase in the percentages of $\mathrm{CD}^{+} \mathrm{T}, \mathrm{CD}^{+} \mathrm{T}$ and NK cells in the mouse peripheral blood, further demonstrating the inhibitory effect of oHSV2 on CRLM. Although oHSV2 serves as a potential novel approach for the treatment of CRLM, there remains a need to further verify its efficacy in CRLM models in other CRC cell lines.

\section{Acknowledgements}

Not applicable.

\section{Funding}

The present study was supported by National Natural Science Foundation of China (grant no. 81650016) and CAMS Innovation Fund for Medical Sciences (CIFMS) (grant no. 2016-I2M-1-001).

\section{Availability of data and materials}

The analyzed data sets generated during the study are available from the corresponding author, on reasonable request.

\section{Authors' contributions}

SL, WZ, QG and BL conceived and designed the experiments. FW, WZ and XH performed the experiments. FW and WZ analyzed the data. FW and WZ wrote the manuscript. JL and $\mathrm{XH}$ assisted with histological analysis. FW, WZ, XH, and JL participated in animal experiments and sample collection.

\section{Ethics approval and consent to participate}

The study protocol was approved by the Ethics of Animal Experiments Committee of the Chinese Academy of Medical Sciences and Peking Union Medical College.

\section{Patient consent for publication}

Not applicable.

\section{Competing interests}

The authors declare that they have no competing interests.

\section{References}

1. Siegel RL, Miller KD and Jemal A: Cancer statistics. CA Cancer J Clin 67: 7-30, 2017.

2. Goss PE, Strasser-Weippl K, Lee-Bychkovsky BL, Fan L, Li J, Chavarri-Guerra Y,Liedke PE,Pramesh CS, Badovinac-Crnjevic T, Sheikine Y, et al: Challenges to effective cancer control in China, India and Russia. Lancet Oncol 15: 489-538, 2014.

3. Varghese $\mathrm{C}$ and Shin HR: Strengthening cancer control in China. Lancet Oncol 15: 484-485, 2014.

4. Adam R: Chemotherapy and surgery: New perspectives on the treatment of unresectable liver metastases. Ann Oncol 14 (Suppl 2): ii13-ii16, 2003.

5. Salah S, Ardissone F, Gonzalez M, Gervaz P, Riquet M, Watanabe K, Zabaleta J, Al-Rimawi D, Toubasi S, Massad E, et al: Pulmonary metastasectomy in colorectal cancer patients with previously resected liver metastasis: Pooled analysis. Ann Surg Oncol 22: 1844-1850, 2015.

6. Van den Eynde M and Hendlisz A: Treatment of colorectal liver metastases: A review. Rev Recent Clin Trials 4: 56-62, 2009.

7. Maffione AM, Lopci E, Bluemel C, Giammarile F, Herrmann K and Rubello D: Diagnostic accuracy and impact on management of (18)F-FDG PET and PET/CT in colorectal liver metastasis: A meta-analysis and systematic review. Eur J Nucl Med Mol Imaging 42: 152-163, 2015

8. Tomlinson JS, Jarnagin WR, DeMatteo RP, Fong Y, Kornprat P, Gonen M, Kemeny N, Brennan MF, Blumgart LH and D'Angelica M: Actual 10-year survival after resection of colorectal liver metastases defines cure. J Clin Oncol 25: 4575-4580, 2007. 
9. Fong Y, Fortner J, Sun RL, Brennan MF and Blumgart LH: Clinical score for predicting recurrence after hepatic resection for metastatic colorectal cancer: Analysis of 1001 consecutive cases. Ann Surg 230: 309-321, 1999.

10. House MG, Kemeny NE, Gonen M, Fong Y, Allen PJ, Paty PB, DeMatteo RP, Blumgart LH, Jarnagin WR and D'Angelica MI: Comparison of adjuvant systemic chemotherapy with or without hepatic arterial infusional chemotherapy after hepatic resection for metastatic colorectal cancer. Ann Surg 254: 851-856, 2011.

11. Kobuchi S, Ito Y, Hayakawa T, Nishimura A, Shibata N, Takada K and Sakaeda T: Pharmacokinetic-pharmacodynamic (PK-PD) modeling and simulation of 5-fluorouracil for erythropenia in rats. J Pharmacol Toxicol Methods 70: 134-144, 2014.

12. Fu X, Tao L, Cai R, Prigge J and Zhang X: A mutant type 2 herpes simplex virus deleted for the protein kinase domain of the ICP10 gene is a potent oncolytic virus. Mol Ther 13: 882-890, 2006.

13. Anzivino E, Fioriti D, Mischitelli M, Bellizzi A, Barucca V, Chiarini $F$ and Pietropaolo V: Herpes simplex virus infection in pregnancy and in neonate: Status of art of epidemiology, diagnosis, therapy and prevention. Virol J 6: 40, 2009.

14. Zhao Q, Zhang W, Ning Z, Zhuang X, Lu H, Liang J, Li J, Zhang Y, Dong Y, Zhang Y, et al: A novel oncolytic herpes simplex virus type 2 has potent anti-tumor activity. PloS One 9: e93103, 2014.

15. Li H, Dutuor A, Fu X and Zhang X: Induction of strong antitumor immunity by an HSV-2-based oncolytic virus in a murine mammary tumor model. J Gene Med 9: 161-169, 2007.

16. Fu X, Tao L and Zhang X: An oncolytic virus derived from type 2 herpes simplex virus has potent therapeutic effect against metastatic ovarian cancer. Cancer Gene Ther 14: 480-487, 2007.

17. Workenhe ST, Simmons G, Pol JG, Lichty BD, Halford WP and Mossman KL: Immunogenic HSV-mediated oncolysis shapes the antitumor immune response and contributes to therapeutic efficacy. Mol Ther 22: 123-131, 2014.

18. Bollino D, Colunga A, Li B and Aurelian L: $\triangle \mathrm{PK}$ oncolytic activity includes modulation of the tumor cell milieu. J Gen Virol 97: 496-508, 2016.

19. Colunga AG, Laing JM and Aurelian L: The HSV-2 mutant DeltaPK induces melanoma oncolysis through nonredundant death programs and associated with autophagy and pyroptosis proteins. Gene Ther 17: 315-327, 2010.

20. Yang H, Peng T, Li J, Wang Y, Zhang W, Zhang P, Peng S, Du T, Li Y, Yan Q and Liu B: Treatment of colon cancer with oncolytic herpes simplex virus in preclinical models. Gene Ther 23: 450-459, 2016.

21. Kozlowski JM, Fidler IJ, Campbell D, Xu ZL, Kaighn ME and Hart IR: Metastatic behavior of human tumor cell lines grown in the nude mouse. Cancer Res 44: 3522-3529, 1984.

22. Yin L, Zhao C, Han J, Li Z, Zhen Y, Xiao R, Xu Z and Sun Y: Antitumor effects of oncolytic herpes simplex virus type 2 against colorectal cancer in vitro and in vivo. Ther Clin Risk Manag 13: 117-130, 2017.

23. Yang H, Peng T, Li J, Wang Y, Zhang W, Zhang P, Peng S, Du T, Li Y, Yan Q and Liu B: Treatment of colon cancer with oncolytic herpes simplex virus in preclinical models. Gene Ther 23: 450-459, 2016.

24. Fu X, Rivera A, Tao L and Zhang X: An HSV-2 based oncolytic virus can function as an attractant to guide migration of adoptively transferred T cells to tumor sites. Oncotarget 6: 902-914, 2015.

25. Toda M, Rabkin SD, Kojima H and Martuza RL: Herpes simplex virus as an in situ cancer vaccine for the induction of specific anti-tumor immunity. Hum Gene Ther 10: 385-393, 1999.

26. Ottolino-Perry K, Acuna SA, Angarita FA, Sellers C, Zerhouni S Tang $\mathrm{N}$ and McCart JA: Oncolytic vaccinia virus synergizes with irinotecan in colorectal cancer. Mol Oncol 9: 1539-1552, 2015.
27. Esin E and Yalcin S: Maintenance strategy in metastatic colorectal cancer: A systematic review. Cancer Treat Rev 42: 82-90, 2016.

28. Russell SJ, Peng KW and Bell JC: Oncolytic virotherapy. Nat Biotechnol 30: 658-670, 2012.

29. Sze DY, Reid TR and Rose SC: Oncolytic virotherapy. J Vasc Interv Radiol 24: 1115-1122, 2013.

30. Pol J, Kroemer G and Galluzzi L: First oncolytic virus approved for melanoma immunotherapy. Oncoimmunology 5: e1115641, 2015.

31. Shinozaki K, Ebert O and Woo SL: Eradication of advanced hepatocellular carcinoma in rats via repeated hepatic arterial infusions of recombinant VSV. Hepatology 41: 196-203, 2005.

32. Fong Y, Kim T, Bhargava A, Schwartz L, Brown K, Brody L, Covey A, Karrasch M, Getrajdman G, Mescheder A, et al: A herpes oncolytic virus can be delivered via the vasculature to produce biologic changes in human colorectal cancer. Mol Ther 17: 389-394, 2009.

33. Toda M, Rabkin SD, Kojima H and Martuza RL: Herpes simplex virus as an in situ cancer vaccine for the induction of specific anti-tumor immunity. Hum Gene Ther 10: 385-393, 1999.

34. Shi Y, Liu CH, Roberts AI, Das J, Xu G, Ren G, Zhang Y, Zhang L, Yuan ZR, Tan HS, et al: Granulocyte-macrophage colony-stimulating factor (GM-CSF) and T-cell responses: What we do and don't know. Cell Res 16: 126-133, 2006.

35. Croxford AL, Spath S and Becher B: GM-CSF in neuroinflammation: Licensing myeloid cells for tissue damage. Trends Immunol 36: 651-662, 2015.

36. Cerwenka A, Baron JL and Lanier LL: Ectopic expression of retinoic acid early inducible-1 gene (RAE-1) permits natural killer cell-mediated rejection of a MHC class I-bearing tumor in vivo. Proc Natl Acad Sci USA 98: 11521-11526, 2001.

37. Diefenbach A, Jensen ER, Jamieson AM and Raulet DH: Rae1 and H60 ligands of the NKG2D receptor stimulate tumor immunity. Nature 413: 165-171, 2001.

38. Galon J, Costes A, Sanchez-Cabo F, Kirilovsky A, Mlecnik B, Lagorce-Pages C, Tosolini M, Camus M, Berger A, Wind P, et al: Type, density and location of immune cells within human colorectal tumors predict clinical outcome. Science 313: 1960-1964, 2006.

39. Pancione M, Giordano G, Remo A, Febbraro A, Sabatino L, Manfrin E, Ceccarelli M and Colantuoni V: Immune escape mechanisms in colorectal cancer pathogenesis and liver metastasis. J Immunol Res 2014: 686879, 2014.

40. Alpizar YA, Chain B, Collins MK, Greenwood J, Katz D, Stauss HJ and Mitchison NA: Ten years of progress in vaccination against cancer: The need to counteract cancer evasion by dual targeting in future therapies. Cancer Immunol Immunother 60: 1127-1135, 2011.

41. Seebauer CT, Brunner S, Glockzin G, Piso P, Ruemmele P, Schlitt HJ, Geissler EK, Fichtner-Feigl S and Kesselring R: Peritoneal carcinomatosis of colorectal cancer is characterized by structural and functional reorganization of the tumor microenvironment inducing senescence and proliferation arrest in cancer cells. Oncoimmunology 5: e1242543, 2016.

42. Workenhe ST, Simmons G, Pol JG, Lichty BD, Halford WP and Mossman KL: Immunogenic HSV-mediated oncolysis shapes the antitumor immune response and contributes to therapeutic efficacy. Mol Ther 22: 123-131, 2014.

This work is licensed under a Creative Commons Attribution-NonCommercial-NoDerivatives 4.0 International (CC BY-NC-ND 4.0) License. 